\title{
Dificuldades de adesão ao tratamento na hipertensão arterial sistêmica: considerações a partir de um estudo qualitativo em uma unidade de Atenção Primária à Saúde
}

\section{Difficulties in high blood pressure treatment compliance: considerations based on a qualitative study in a primary health care unit}

\author{
Angélica Manfroi ${ }^{1}$ \\ Francisco Arsego de Oliveira²
}

\section{RESUMO}

A hipertensão arterial sistêmica (HAS) é uma doença crônica, cujo controle é essencial para a prevenção de complicações, em longo prazo, relacionadas à morbidade e à mortalidade cardiovascular e cerebral, dentre outras. O tratamento da HAS baseia-se em medidas não-farmacológicas e farmacológicas. Considerase adesão a um tratamento o grau de coincidência entre a orientação médica e o comportamento do paciente. Na Unidade de Saúde Parque dos Maias, observa-se a dificuldade na manutenção da pressão arterial dos hipertensos, de forma continuada, que pode estar relacionada à falta de adesão destes pacientes ao tratamento. O objetivo do estudo é avaliar os fatores envolvidos na dificuldade de adesão ao tratamento anti-hipertensivo sob o ponto de vista do paciente. Para isso, partimos da pesquisa qualitativa, com entrevistas abertas e semi-estruturadas, individuais, com 13 pacientes adultos hipertensos, inscritos no Programa de Hipertensos da Unidade de Saúde Parque dos Maias. Como resultado, verificamos questões que dificultam a adesão ao tratamento: a) fase inicial assintomática; b) uso de medicamento somente quando pensam que a pressão está elevada (relacionam o aumento a sintomas que crêem ser ligados à HAS, como cefaléia, náuseas, ou quando "ficam nervosos"); c) impressão de cura com conseqüente abandono dos fármacos, quando, na realidade, a pressão está controlada; d) desgosto de ter de tomar remédios continuamente, de ser "dependentes" deles; e) sintomas adversos dos fármacos como disfunção erétil e tosse; f) dieta hipossódica é difícil de ser seguida, principalmente pelo fato de os familiares terem de se habituar a ela; g) necessidade de consultas médicas mensais para fornecimento de prescrições para a retirada do medicamento na unidade de saúde; h) falta de medicamento gratuito na unidade de saúde, em algumas instâncias; i) alguns pacientes ficam "escravos" dos horários da ministração de medicamentos, o que dificulta sua rotina diária. A conclusão

\section{PALAVRAS-CHAVE:}

- Hipertensão;

- Atenção Primária à Saúde;

- Pesquisa Qualitativa.

\section{KEY-WORDS:}

- Hypertension;

- Primary Health Care;

- Qualitative research.

${ }^{1}$ Médica de Família e Comunidade, Secretaria Municipal de Saúde de Florianópolis, SC, Brasil.

${ }^{2}$ Médico de Família e Comunidade, Serviço de Saúde Comunitária - Grupo Hospitalar Conceição, Departamento de Medicina Social, Faculdade de Medicina, Universidade Federal do Rio Grande do Sul, Porto Alegre, Brasil. 
é que é muito importante a equipe de saúde conhecer as dificuldades dos pacientes em aderir ao tratamento anti-hipertensivo com o objetivo de tentar corrigilas, juntamente com o paciente, para melhor controle da HAS. Enfatiza-se, principalmente, a importância da comunicação no relacionamento médico/equipepaciente, o que envolve a confiança e, por conseguinte, possibilidades de maior adesão ao tratamento.

\section{ABSTRACT}

Introduction: High blood pressure $(H B P)$ is a chronic disease whose control is essential for preventing long-term complications related to cardiovascular mortality and morbidity. The treatment for HBP is based on non-pharmacological and pharmacological measures. Treatment compliance is characterized by the degree of coincidence between medical orientation and patient behavior. On Primary Health Care level there are often difficulties in maintaining a systematic control over the blood pressure of hypertensive patients, probably due to the lack of treatment compliance of these patients. Objective: Assess the factors involved in the difficulty of compliance with anti-hypertensive treatment from the patients' point of view. Methodology: Qualitative research, with open and semi structured individual interviews with 13 hypertensive adults, enrolled in the Program for Hypertensive Patients of a Primary Health Care Unit in Porto Alegre, Brazil. Results: Factors hampering treatment compliance were: a) initial asymptomatic phase; $b$ ) use of medication only when patients think their blood pressure is high (the increase is associated with symptoms patients believe to be a consequence of HBP such as headache, nausea, or when they "get nervous"); c) the idea of cure and abandonment of treatment while, in fact, their blood pressure is but under control; d) resistance in taking the medication in a systematic way, making them "dependent"; e) side effects of the drugs, such as erectile dysfunction and coughing ; f) difficulty in following a hyposodic diet, besides the fact that the relatives have to get used to it as well; $g$ ) the need for monthly appointments in order to obtain a refill of the medication at the health care unit: $h$ ) lack of free medication at the health care unit; i) some patients feel they are "slaves" of the schedule they have to follow because it is interfering with their daily routine. Conclusion: It is important that the health care professionals know the patients' difficulties to be compliant with anti-hypertensive treatment so they can try correcting the problems together with the patient for a better control of HBP. It has to be pointed out that the trust resulting from a good doctor/patient relationship contributes greatly to a better treatment compliance of the patient.

\section{Introdução}

Não há dúvidas de que, atualmente, a hipertensão arterial sistêmica (HAS) constitui um sério problema de saúde pública em todo o mundo. A HAS é comprovadamente um fator de risco para uma série de outras doenças e agravos à saúde, sendo, portanto, considerada a origem das doenças crônicodegenerativas ${ }^{1}$. Em relação a dados brasileiros, sua prevalência oscila entre 15 e $20 \%$ na população adulta $^{2}$. Em Porto Alegre (RS), a prevalência de HAS atinge a cifra de $19,2 \%^{3}$.

A HAS é considerada uma doença crônica, com longo curso assintomático, evolução clínica lenta, prolongada e permanente, podendo evoluir para 
situações de complicação, sendo um dos principais fatores de morbidade e mortalidade cardiovascular e cerebrovascular ${ }^{4}$.

Dessa forma, a HAS é um problema de saúde pública cujo controle, de forma continuada, é essencial e visa à prevenção de alterações irreversíveis no organismo e relacionadas à morbimortalidade associadas à doença, exigindo, portanto, ações nos níveis individual e coletivo.

O tratamento da HAS baseia-se em medidas não-farmacológicas e farmacológicas. Em relação aos cuidados não-farmacológicos, os objetivos referem-se a mudanças no estilo de vida, incluindo os cuidados com dieta com restrição de sal, redução de peso, atividade física regular, abandono do tabagismo e do álcool. Dentre as medidas farmacológicas, há inúmeras classes de anti-hipertensivos disponíveis, variando o seu mecanismo de ação, a sua potência, posologia e efeitos adversos ${ }^{5}$.

Em que pese o grande avanço científico e tecnológico no manejo da hipertensão arterial ocorrido nos últimos anos, uma das grandes dificuldades atuais refere-se à adesão dos pacientes aos tratamentos instituídos, ou seja, até que ponto o paciente segue as recomendações dadas pelo médico ou outro profissional de saúde para o controle do seu problema de saúde. Considera-se adesão a um tratamento o grau de coincidência entre a prescrição médica - o que inclui as orientações nãofarmacológicas - e o comportamento adotado concretamente pelo paciente. No caso da HAS, envolve a extensão em que o comportamento do indivíduo (em termos de uso efetivo do medicamento, realização de mudanças no estilo de vida e comparecimento às consultas médicas) coincide com o conselho médico ${ }^{6}$. Assim, o controle inadequado da pressão arterial pode estar relacionado à falta de adesão do paciente hipertenso ao tratamento indicado.

A adesão do paciente a uma determinada terapia depende de vários fatores que incluem, dentre outros, os relativos à relação médico-paciente, às questões subjetivas do paciente, às questões referentes ao tratamento, à doença, ao acesso ao serviço de saúde, à obtenção do medicamento prescrito e à continuidade do tratamento ${ }^{7}$. Neste sentido, é de fundamental importância que o médico esclareça, continuadamente e em linguagem acessível ao nível de compreensão do paciente, conceitos básicos quanto ao significado da HAS, sua etiologia, evolução, conseqüências, cuidados necessários, fármacos utilizados e seus potenciais efeitos colaterais. Além disso, é importante que haja vínculo suficiente entre médico e paciente, para que este se sinta engajado no seu tratamento. Uma vez que o paciente se sinta esclarecido sobre sua doença, e que se estabeleça o elo médico-paciente, o paciente tende a assumir responsabilidade pelos cuidados com sua saúde, juntamente com o médico ${ }^{8}$.

Além da relação médico-paciente, deve-se considerar, também como fator importante, que os pacientes hipertensos experimentam a influência de variados determinantes de adaptação às doenças crônicas que dependem da característica de personalidade do indivíduo, dos seus mecanismos de enfrentamento de problemas, do seu autoconceito, da sua auto-imagem e da sua auto-estima, da experiência prévia com a doença e/ou doenças e, ainda, das atitudes dos cuidadores da área de saúde. As respostas emocionais dos pacientes devem ser consideradas, já que, muitas vezes, está presente o mecanismo de regressão, em que o paciente mani- 
festa um comportamento infantilizado, apresentandose emocionalmente dependente na realização de suas atividades, nas quais se incluem, principalmente, os cuidados com sua saúde? .

Sendo a HAS uma doença sem manifestações clínicas, pelo menos precocemente, os pacientes também podem apresentar sentimentos naturais de negação frente à doença, com uma conseqüente não-adesão ao tratamento antihipertensivo. Isto é, torna-se perfeitamente compreensivel que um paciente que não se sente "doente", sob o ponto de vista estritamente biomédico, evite o uso de medicamentos ${ }^{9}$.

Neste mesmo sentido, como a HAS é uma doença assintomática até que as complicações evidenciem-se em longo prazo, os pacientes podem não perceber a importância de manter um tratamento continuado. Além disso, as mudanças de estilo de vida requerem dedicação e persistência. Muitos fármacos, por sua vez, apresentam efeitos adversos, exigem horários especiais para as ingestões diárias, significam custo adicional no orçamento doméstico, dentre outros inúmeros fatores que podem dificultar o seguimento do tratamento da HAS por qualquer pessoa.

Pelo fato de a HAS ser uma doença crônica que exige cuidado continuado, dependendo desse somatório de fatores e da relação entre eles, a terapia proposta pode ter êxito completo ao que se propõe ou fracassar parcial ou completamente.

Buscando explorar de forma mais aprofundada essas questões no âmbito da Atenção Primária à Saúde (APS), foi realizada uma pesquisa de caráter qualitativo junto à Unidade de Saúde Parque dos Maias, em Porto Alegre (RS). A Unidade de Saúde Parque dos Maias integra o Serviço de
Saúde Comunitária (SSC) do Grupo Hospitalar Conceição (GHC), um complexo de saúde vinculado ao Ministério da Saúde, que oferece atenção à saúde nos níveis primário, secundário e terciário. Em relação à APS, o Serviço de Saúde Comunitária é composto de 12 Unidades Básicas de Saúde, situadas na zona norte da cidade de Porto Alegre, cobrindo uma população de mais de 120 mil habitantes. A Unidade de Saúde Parque dos Maias presta atendimento, desde 1992, a uma população composta por 2.137 famílias cadastradas, perfazendo 8.482 pessoas. Deste total, a população adulta compreende 4.535 pacientes, tendo 327 hipertensos cadastrados.

É freqüente, nessa unidade de saúde, o relato da equipe sobre a dificuldade de se manter a pressão arterial dos pacientes hipertensos em níveis aceitáveis, de forma continuada, até mesmo entre os pacientes que se consultam regularmente. Uma das explicações para esse problema pode ser a falta de adesão dos pacientes ao tratamento proposto.

Dessa forma, este trabalho teve como objetivo avaliar os fatores envolvidos na dificuldade de adesão ao tratamento anti-hipertensivo (medidas não farmacológicas) sob o ponto de vista do paciente. Assim, buscou-se entender os fatores que dificultam o seguimento adequado das orientações fornecidas pelo médico e demais integrantes da equipe de saúde.

\section{Metodologia}

Realizou-se uma pesquisa qualitativa, cujos focos principais eram as entrevistas com os pacientes moradores do bairro Rubem Berta, Vila Parque dos Maias I, pertencentes à área de atendimento da Unidade de Saúde Parque dos Maias e inscritos no Programa de Hipertensos do SSC/GHC.

O Programa de Hipertensos desenvolvido na 
unidade tem como objetivo o acompanhamento sistematizado dos pacientes hipertensos, visando ao manejo adequado da HAS. As atividades previstas no programa são: o cadastro dos pacientes, a distribuição de medicamentos e o atendimento individual ou em grupo mensal. Nesse Programa, estão incluídos pacientes adultos hipertensos de ambos os sexos, de diferentes raças e variados níveis de formação educacional, crenças religiosas e situação conjugal. No presente estudo, não foram incluídos pacientes pediátricos, pacientes com hipertensão secundária nem gestantes com doença hipertensiva específica da gestação. Também foram desconsiderados pacientes com outras doenças crônicas que requeiram modificações no estilo de vida e uso de fármacos de forma continuada. O estudo foi divulgado para todos os pacientes hipertensos da Unidade. A participação dos mesmos ocorreu de forma espontânea, com assinatura de termo livre-esclarecido.

Para a coleta das informações foram feitas entrevistas individuais pela autora a pacientes específicos, com duração média de 30 minutos, na própria Unidade de Saúde. Ao final do estudo, foram entrevistados 13 pacientes. A técnica utilizada nas entrevistas foi a de entrevista aberta, conforme metodologia de Minayo ${ }^{9}$, somada a um roteiro semiestruturado, a partir de um modelo citado na literatura $^{10}$.

No decorrer das entrevistas, procurava-se abordar questões relativas ao entendimento do paciente a respeito da HAS, sua experiência prévia com a doença, as formas de controle, a noção da cronicidade da doença e a potencial lesão em órgãosalvo. Discutia-se, além disso, o seu conhecimento acerca dos tipos de tratamento existentes, a sua utilização, bem como as dificuldades encontradas no tratamento anti-hipertensivo como um todo. As entrevistas foram gravadas em fita cassete, após ter sido obtido o consentimento pós-informação do paciente para participação na pesquisa. O projeto de pesquisa foi aprovado pelo Comitê de Ética em Pesquisa do Grupo Hospitalar Conceição.

\section{Resultados}

Do total de 13 entrevistados, dez eram do sexo feminino e três do sexo masculino. As idades variaram de 34 a 70 anos. Grande parte desses informantes não possuia o ensino fundamental completo, não havendo pacientes analfabetos. A maioria dos pacientes não exercia atividade remunerada atualmente, recebendo auxílio previdenciário em torno de um salário mínimo. Uma única paciente não reclamou da sua situação financeira atual, pois recebia de três a quatro salários mínimos mensais, somando-se o auxílio previdenciário à atividade de cozinheira em um restaurante. Apenas dois pacientes moravam sozinhos. Dos 13 entrevistados, cinco participaram, pelo menos uma vez, da atividade em grupo do Programa de $\mathrm{Hi}-$ pertensos, sendo uma delas freqüentadora assídua dessa atividade.

Em uma perspectiva inicial, chamou atenção o fato de que os pacientes entrevistados demonstraram desconhecimento sobre o que significa "ter hipertensão". Muitos não sabiam tratar-se de uma doença. Mesmo assim, pareciam estar cientes da importância de realizar algum tipo de cuidado para manter a pressão arterial controlada, como neste depoimento de uma paciente de 70 anos:

"Eu não sei o que é [HAS], não sei se é doença. Às vezes, eu penso que é até um sentimento, uma coisa que se altera com os problemas da pessoa, assim, um mal-estar, acho que não chega 


\section{a ser doença."}

É importante comentar a questão da ausência de sintomas da HAS, por longo período, até que, se não tratada adequadamente, comecem a surgir as complicações decorrentes da doença. Devido à ausência de sintomas, vários pacientes entrevistados referiram que abandonaram o tratamento. Retomaramno após uma crise hipertensiva, alguma complicação decorrente da HAS ou devido a outro problema de saúde em que o monitoramento da pressão se fez presente. Outros informantes, mesmo assintomáticos e sem apresentar nenhuma complicação decorrente da HAS, acreditam ser importante manter o tratamento adequado, principalmente como prevenção de complicações a longo prazo. Esses pacientes assintomáticos informaram que "não se sentem doentes", por mais que tenham de tomar os medicamentos de forma continuada. Há, por outro lado, os casos em que os pacientes sentem-se doentes justamente depois de iniciado o tratamento anti-hipertensivo. Isso se explica pelo fato de que, antes de se saberem hipertensos, esses pacientes tinham suas vidas livres de cuidados e do uso de medicamentos. A partir do diagnóstico de HAS, entretanto, necessitaram usar medicamentos de forma contínua e buscar um estilo de vida mais saudável. O fato de terem de usar medicamentos é que os fez se sentirem doentes, como é exemplificado pelo comentário de um paciente de 63 anos:

"Antes de ficar hipertenso, eu não tinha nenhum problema de saúde. Foi saber que minha pressão é alta, que tudo começou a complicar. Tenho que tomar remédio, sempre, todos os dias. Isso me lembra, todo dia, que sou doente."

Também há os que eram assintomáticos até o diagnóstico de HAS feito pelo médico. Quando iniciaram o uso de medicamentos, passaram a apresentar efeitos adversos, o que fez esses pacientes sentirem-se, a partir de então, "doentes". Na realidade, os sintomas do "sentir-se doente", neste caso, são os relativos aos efeitos colaterais dos medicamentos e não à HAS em si. Nessa situação, o fato de estarem usando remédio devido à HAS significa que, se há sintomas, estes são devidos à doença recémdiagnosticada.

"Logo [após o diagnóstico de HAS] comeceia usar remédio. Depois, eu parei uns quatro meses. Não sentia nada antes, para que tomar, se me sentia fraca tomando? Achava que iria dar certo, assim, sem remédio, mas não adiantou, vim aqui, estava com a pressão alta de novo... Voltei a tratar."

Uma questão recorrente em alguns relatos foi o que podemos chamar de "sonho de cura da HAS". Este desejo, na realidade, apareceu de forma um tanto contraditória, em algumas respostas. Mesmo os pacientes que referiram saber ter de usar medicamentos continuamente, cientes de não haver cura da HAS, em algum momento da entrevista demonstraram vontade de "um dia não precisar mais usar medicamentos" para a HAS, devido justamente à sua cura:

"Ah, mas eu imagino... Estou com essa idade, mas imagino que um dia vou parar de tomar remédio para a pressão. Imagino o médico me dizendo que fiquei curada."

Há também os que acreditam que "curaram" a doença no momento em que atingem o controle adequado dos níveis de pressão arterial com o uso do tratamento prescrito. Assim, muitos pacientes abandonam o tratamento farmacológico nesse momento, ao invés de mantê-lo e seguir com o sucesso no controle da HAS. Soma-se a isso o fato de esses 
pacientes, muitas vezes, estarem previamente assintomáticos:

"Eu estava com a pressão boa, não sentia nada, parei de tomar os remédios. Para que tomar se não tinha nada?"

Alguns pacientes referem sintomas específicos, como dor de cabeça e tonturas, quando julgam estar com a pressão arterial elevada. É importante ressaltar que os pacientes que referiram algum sintoma, relacionando-o como conseqüência de pressão arterial elevada, não a mediram para conferir se, de fato, estava alterada, na vigência dos sintomas referidos. Além de relacionarem seus sintomas com aumento da pressão, não questionam que estes possam estar relacionados a outras causas, dentre elas os efeitos colaterais dos fármacos utilizados para tratamento da pressão arterial. Neste sentido, a partir do momento em que o diagnóstico está consolidado, parece haver uma ênfase em atribuir à pressão alta qualquer sintoma apresentado pelo paciente.

Por outro lado, os pacientes demonstraram ter uma boa noção das complicações cardiovasculares e cerebrovasculares relacionadas à HAS. Uma das motivações para seguir o tratamento é o medo de ter um acidente vascular cerebral (AVC) ou infarto agudo do miocárdio, as complicações cardiovasculares mais temidas pelos pacientes e atribuídas à HAS.

"Se eu não tratasse, eu já teria morrido, teria sofrido alguma coisa. Acho que a pessoa tem um limite. Quando fui parar no hospital, eu cheguei ao meu limite. A pressão estava altíssima, e se a pessoa não faz tratamento, assim, certinho, acho que não tem como agüentar, o coração começa a disparar, parece que a gente está subindo nas nuvens, pode dar derrame, enfarte."

Há alguns fatores que os pacientes relaciona- ram ao controle/descontrole da pressão arterial e dificuldades ou não em seguir o que consideram correto fazer. Na grande maioria dos relatos, apareceu o "problema de nervos" como sendo o responsável pelo aumento da pressão arterial. Apesar de não ter sido explicitado exatamente nestes termos em nossa pesquisa, há relatos na literatura que relacionam a própria expressão "hipertensão" a um estado emocional em que o indivíduo estaria "muito tenso" 11. Além disso, os informantes também referem o uso inadequado dos medicamentos, a dieta com muito sal (relacionam a dieta rica em gorduras como responsável por alterar a pressão arterial), o tabagismo, a ingestão de bebidas alcoólicas e as dores em geral. Aparece aqui o sentimento de "missão cumprida" quando se sentem cumprindo aquilo que julgam ser "o correto" e, juntamente, a sensação de controle da pressão arterial, bem como dos sintomas atribuídos ao aumento da pressão arterial. Por outro lado, o contrário também ocorre, ou seja, o sentimento de culpa caso não sigam as recomendações médicas.

"Antes [do diagnóstico], era liberado tudo assim. Não tinha preocupação em ter que ficar me cuidando. Depois, é tomar remédio para cuidar da pressão, é parar com sal, cafezinho, cigarro, coisas que eu estava sempre fazendo. Agora é tentar diminuir. Assim, os remédios ajudam a cuidar e lutam contra as outras coisas que fazem mal, entende? Só que as coisas que fazem mal eu estava acostumada, gosto de fazer, então complica, né?"

Foi interessante notar que, mesmo quando seguiam as orientações médicas, tais como modificações no estilo de vida, uso correto de medicamentos, consultas e aferições da pressão arterial freqüentes, alguns pacientes demonstraram insatis- 
fação com o descontrole dos seus níveis tensionais. Esse sentimento pode ser melhor exemplificado com o depoimento de uma informante de 70 anos:

"Meu Deus, eu faço tudo tão direitinho, então por que tem dia que ela está 13 por 7 , hoje está 15 por 8. Então é com isso aí que eu me irrito, e às vez parece que quanto mais se mede a pressão, mais sobe, mais fica sem aquele controle. Então eu fico irritada com isso aí."

Outra questão que chamou atenção é a percepção de que há uma pressão certa para cada paciente: "o meu normal" de pressão arterial. Os pacientes se acostumam com certos valores que se repetem e acreditam ser este o seu padrão normal de pressão arterial, mesmo conhecendo os valores ideais. Fica, então, a questão do papel dos profissionais de saúde, principalmente o do médico, na educação do paciente e no ajuste adequado dos medicamentos, visando ao melhor controle da pressão arterial desses pacientes. É comum observar que muitos médicos deixam-se influenciar por este padrão próprio estabelecido pelos pacientes, tolerando níveis mais elevados da tensão arterial.

Mesmo tendo a consciência da importância do uso do medicamento para controle da HAS, alguns pacientes demonstraram inconformidade em ter de "depender do remédio". O fato de terem de tomar um remédio continuamente, como em qualquer tratamento de longo prazo, visando à manutenção da saúde, gera insatisfação. Melhor seria, segundo esses pacientes, "não ter de tomar nenhum medicamento". Soma-se a isso, mais uma vez, a questão da ausência de sintomas. Isto é, parece realmente incompreensível ficar dependente de medicamentos para tratar uma "doença" que não apresenta nenhum sintoma. Em um estudo qualitativo com o objetivo de avaliar a decisão dos pacientes hipertensos em tomar ou não os medicamentos, Benson e Britten apontam esta questão como importante, na qual se enfatiza a resistência ao uso de medicamentos de uma maneira geral, o que se reflete, também, no uso dos anti-hipertensivos $^{12}$. A fala de uma informante de 64 anos nos ajuda a entender essa questão:

"Eu gostaria de não depender do remédio, mas fazer o quê? E, na minha idade, a tendência é a gente ir tomando cada vez mais remédios. Com a idade, as coisas começam a aparecer. Por tudo isso, estou me cuidando agora, para não ficar uma velha cheia de coisas."

Quando nos referimos a tratamentos de uso contínuo, não podemos esquecer a questão dos efeitos adversos dos medicamentos como um motivo importante para o abandono do tratamento. Dois entrevistados relataram que quase abandonaram o tratamento devido a efeitos indesejáveis provocados pelos fármacos. Porém, após a troca destes, os pacientes sentiram-se motivados a seguir o tratamento, uma vez que os sintomas adversos foram suprimidos.

Uma vez que o esquecimento de tomar a medicação pode ser um fator importante na dificuldade de adesão ao tratamento, tentou-se abordar esta questão durante as entrevistas. Contrariamente à nossa idéia inicial, os informantes não pareceram ter dificuldades em lembrar de tomar os remédios. Quando percebem a importância de se tratar, os medicamentos passam a ser parte de sua rotina. Também é importante ressaltar os horários prescritos pelos médicos, o que, em algumas situações, pode dificultar o dia-a-dia dos pacientes, principalmente se estes não sabem como adaptar os horários de uma forma adequada e cômoda. 
"Eu não esqueço não. Só que antes tomava de manhã, e agora mudou para de 8 em 8 horas, e isso ficou ruim, eu quase esqueci de tomaro da tarde, esses dias. Teve outro dia que eu estava cansada, queria tirar uma sesta, mas estava preocupada com o horário do remédio, dormi olhando para o relógio para não esquecer."

Em se tratando de uma população de baixa renda, é importante que os medicamentos sejam fornecidos gratuitamente, uma vez que a necessidade de compra destes pode interferir negativamente no orçamento doméstico e, assim, ser um fator de nãoadesão ao tratamento. Alguns pacientes também relacionam à baixa renda a dificuldade de manter uma "dieta saudável" conforme prescrição médica. Além disso, surgiu o problema da chamada "peregrinação" a outras unidades de saúde para se tentar conseguir um determinado medicamento, quando este estava em falta na unidade de origem. Percebe-se, então, que tal "peregrinação" é uma constante na vida desses pacientes, em sua busca por medicamento gratuito, considerado um direito, que em princípio deveria ser disponibilizado pelo município, para todas as unidades de saúde; porém, na prática, muitas vezes, isso não ocorre.

"Quando a gente vai à farmácia [da unidade de saúde] pegar e não tem. Aí, vai à outra [unidade de saúde] e também não tem. Semana passada eu consultei, não tinha o remédio, o rapaz disse para vir na segunda-feira, não tinha chegado. Daí, tive de comprar duas vezes o captopril, isso não pode ser assim."

Na Unidade de Saúde Parque dos Maias, a prescrição dos medicamentos anti-hipertensivos, na sua maioria, tem validade mensal. Dessa forma, os pacientes consultam mensalmente para revisão com os médicos e para renovação das prescrições. Nos casos em que o paciente está com sua pressão sob controle, alguns médicos fazem prescrições para dois ou três meses. Percebemos que essa sistemática gera satisfação para o paciente, que se sente adequadamente atendido. Porém, há casos em que o paciente necessita de consulta mensal para obter as receitas, ou porque o médico assim o prefere, mesmo sendo o caso de um paciente com pressão sob controle, ou porque a pressão está continuamente "descontrolada". Nessa situação, há certa insatisfação por parte do paciente, pois é ele que tem de marcar consulta sempre que os fármacos estão por acabar, mesmo havendo facilidade na marcação de consultas para as pessoas vinculadas ao grupo de hipertensos. Em muitos casos, os pacientes saem de uma consulta com o seu retorno no mês seguinte agendado. Mesmo havendo a facilidade de acesso para esses pacientes, o fato de terem de se consultar mensalmente com o médico, simplesmente para receberem a prescrição, não seria uma forma desestimulante à adesão, muito pelo fato de o paciente ser lembrado, a cada mês, de que "está doente": esse é, na verdade, o motivo da consulta com o médico. Assim, cabe o seguinte questionamento: os pacientes cuja pressão encontra-se sob controle e que necessitam apenas dos medicamentos para a manutenção de sua saúde não deveriam ter mais liberdade "em cuidar-se sem tanta dependência do médico?"

“O problema é que para ti pegar remédio tem que ter receita. Então, eu queria que se agilizasse a receita. Lá no Postão [unidade de referência], dão um tipo de receita que dura seis meses, mas eles dão o primeiro mês, depois tenho que pegar no posto próximo de casa, que é aqui. Por que tem que con- 
sultar todo o mês para contar a mesma coisa, só porque tem que ter receita? Eu acho muita coisa vir todo mês. Minha mãe vai ao grupo de hipertensos do posto onde mora e não precisa nem de receita, eles deram uma ficha para ela, e ela vai sempre ao grupo, eles anotam na ficha os remédios que ela pega."

Acredita-se que a relação médico-paciente é um fator importante, se não fundamental, para a manutenção do tratamento. Nessa relação, faz-se necessário que o médico esteja disponível para o paciente na compreensão de seu processo saúde/ doença, com o objetivo de auxiliá-lo nos cuidados com a sua saúde. Resolvemos expandir esse conceito, aqui, para a relação com a equipe de saúde como um todo e percebemos que os pacientes sentem-se bem atendidos pela equipe da Unidade de Saúde. Fica uma dúvida, no entanto: o sentir-se bem atendido significa ser, de fato, bem orientado pela equipe sobre sua doença e os cuidados necessários para o seu tratamento, ou os pacientes referem-se ao fato de serem tratados com respeito e cordialidade enquanto usuários da Unidade de Saúde? Além disso, pelo fato de muitos pacientes considerarem os médicos (ou a equipe) os detentores do conhecimento, por mais que a saúde seja sua, e por mais que deva haver uma aliança com objetivos comuns estabelecidos entre ambos para o sucesso terapêutico, parece estabelecer-se uma relação em que o paciente assume uma atitude passiva. Muitos profissionais, obviamente, assumem uma atitude paternalista, de "donos da verdade", direcionando as condutas a serem desempenhadas pelos pacientes. Dessa forma, este tipo de relação médico-paciente faz com que esse último não se sinta responsável por sua saúde, pois essa responsabilidade é transferida para o médico ou a equipe. Tal tipo de relacionamento deve ser enfatizado, pois é muito comum em nosso país, com conseqüências desastrosas no seguimento desses indivíduos. A seguir, um bom exemplo de como a paciente expressa a sua culpa por não ter seguido as orientações do médico:

"Eu sei que fui bem orientada pelos médicos, entendeu? As falhas que aconteceram, no caso, foram minhas mesmo, sabe? Porque eu fui rebelde, não quis fazer [o tratamento]. Aqui no posto, todos são bons para a gente."

Por fim, outra questão que consideramos relevante valorizar foi o acesso aos serviços prestados pela Unidade de Saúde. Como os pacientes atendidos pertencem à sua área de atuação, a maioria deles mora muito perto, o que facilita o acesso, sendo um aspecto facilitador para a adesão ao tratamento antihipertensivo.

\section{Considerações Finais}

Em se tratando da adesão a um tratamento, percebemos que há inúmeras questões envolvidas no sucesso ou no fracasso em obtê-la. Neste trabalho, abordamos algumas das questões que surgiram em entrevistas com alguns pacientes. Evidentemente, a HAS e o seu tratamento adequado envolvem uma multiplicidade de fatores extremamente complexos, que exigem de todos os envolvidos o emprego de estratégias combinadas que dêem conta dessa complexidade $^{13}$. De qualquer forma, ao falarmos de adesão, devemos sempre considerar a subjetividade que faz com que cada indivíduo, de acordo com as suas vivências, conhecimentos, crenças e valores, tenha um comportamento muito próprio em relação ao significado de "sentir-se doente". Isso reflete na forma como esse indivíduo manifesta-se quando 
abordamos essas questões. Da mesma maneira, também não podemos desconsiderar as crenças do médico, ou da equipe de saúde, as quais, muitas vezes, podem não coincidir com as crenças e os interesses do paciente.

Um dos aspectos principais neste tema diz respeito, então, às percepções diferentes em relação à saúde e à doença por parte do médico - e da equipe de saúde - e dos pacientes. Esse é um dos fatores determinantes sobre a "aceitação" ou não do "diagnóstico" de HAS dado pelo médico e o tratamento a ser seguido.

Nesse sentido, como afirma Chockalingam e cols., a educação dos pacientes hipertensos é essencial, mas, em determinadas situações, não será suficiente para fazer com que o paciente siga as recomendações estabelecidas como corretas à luz do conhecimento científico atual ${ }^{14}$.

O que a experiência desta pesquisa aponta é o caminho inverso: não somente educar o paciente, mas iniciar uma abordagem terapêutica que inclua uma negociação sobre o tratamento e o seu seguimento partindo das concepções que o paciente tem a respeito da sua doença. É bom ressaltar que, com isso, não se está negando o conhecimento técnico ou se abandonando as conviç̧ões profissionais. O que se propõe é o estabelecimento de uma parceria entre pacientes e médicos, cujo objetivo maior é o controle adequado da HAS e uma redução nas suas complicações.

Isso pode parecer óbvio à primeira vista, mas acreditamos que os médicos possam estar falhando nesta área. De pouco adianta saber de forma aprofundada a fisiopatologia da HAS e os tratamentos mais modernos disponíveis se não se consegue uma comunicação adequada com os pacientes. E um paciente que não se comunica com o seu médico é um candidato muito forte a abandonar o seu tratamento. Ao longo do tempo, nós, médicos, deixamos de nos comunicar com eles e, sem dúvida, uma comunicação adequada entre médico e paciente melhora o desfecho clínico ${ }^{15}$.

$$
\text { É, portanto, fundamental enfatizar que o foco }
$$

passa, conforme esta visão, do seguimento adequado para a comunicação adequada. E essa é uma mudança radical no sentido de que a responsabilidade do tratamento volta a ser dividida entre médico e paciente. Não bastará, por exemplo, simplesmente prescrever um determinado tratamento e esperar que o paciente "educado" o siga. O médico e toda a equipe de saúde terão mais uma atribuição: a arte da comunicação, pois esta será fundamental no caminho para o sucesso terapêutico, no que diz respeito a toda a sua complexidade, tanto individual como coletivamente.

\section{V.Referências}

1. Fuchs FD. Hipertensão arterial sistêmica. In: Medicina ambulatorial: condutas de atenção primária baseadas em evidências. 3. ed. Porto Alegre (RS): Artmed; 2004. p. 641-56.

2. III Consenso Brasileiro de Hipertensão Arterial. Rev Bras Clin Terap. 1998; 24 (6):231-72.

3. Fuchs FD, Moreira LB, Moraes RS, Bredemeier M, Cardozo SC. Prevalência de hipertensão arterial sistêmica e fatores associados na região urbana de Porto Alegre. Arq Bras Cardiol. 1994; 63:473-9.

4. Lessa I. Doenças crônicas não transmissíveis. In: $O$ adulto brasileiro e as doenças da modernidade: Epidemiologia das doenças não transmissíveis. São Paulo, Rio de Janeiro: Hucitec, Abrasco; 1998. p. 29-42. 5. Joint National Committee on Prevention, Detection, 
Evaluation, and Treatment of High Blood Pressure. The seventh report of the Joint National Committee on Prevention, Detection, Evaluation, and Treatment of High Blood Pressure. JAMA. 2003; 289: (19): 2560-72.

6. Horwitz RI, Horwitz SM. Adherence to the treatment and health outcomes. Arch Intern Med. 1993; 153:1863-8.

7. Chizzola PR, Mansur AJ, Luz PL, Bellotti G. Compliance with pharmacological treatment in outpatients from a brazilian cardiology referral center. São Paulo Med Journal. 1996; 114:1259-64.

8. Unger T. Patient-doctor interactions in hypertension. J Hum Hypertens. 1995: 41-6.

9. Minayo MCS. O desafio do conhecimento: pesquisa qualitativa em saúde. São Paulo: Hucitec; 1993. 269 p. 10. Nobre F, Pierin A, Mion Júnior D. Adesão ao tratamento: o grande desafio da hipertensão. São Paulo: Lemos Editorial; 2001. p. 26.

11. Center for Disease Control and Prevention. Health beliefs and compliance with prescribed medication for hypertension among black women - New Orleans 1985-86. In: Brown Peter J. Understanding and applying medical anthropology. Mountain View: Mayfield; 1998. p. 248-50.

12. Benson J, Britten N. Patient's decisions about whether or not to take antihypertensive drugs: qualitative study. BMJ. 2002; 325; 873-7.

13. Schroeder K, Fahey T, Ebrahim S. How can we improve adherence to blood pressure-lowering medication in ambulatory care?: systematic review of randomized controlled trials. Arch Int Med. 2004; 164 (7):722-32.

14. Chockalingam A et al. Adherence to management of high blood pressure: recommendations of the Canadian Coalition for High Blood Pressure Prevention and Control. Can J Public Health. 1998; 89 (5): 15-7.
15. Van Wieringen, JCM, Harmsen JAM, Bruijnzeels MA. Intercultural communication in general practice. Eur J Public Health. 2002; 12: 63-8.

\section{Endereço para correspondência:}

Angélica Manfroi

Rua Antônio Dias Carneiro, 557

Florianópolis - SC

CEP: $88051-200$

\section{Endereço eletrônico:}

angelica.manfroi@gmail.com 